\title{
Perfil da utilização de antimicrobianos em um hospital privado
}

\author{
The profile of antimicrobial utilization in a private hospital
}

Fernanda d'Athayde Rodrigues ${ }^{1}$

Andréa Dâmaso Bertoldi ${ }^{2}$

\footnotetext{
${ }^{1}$ Instituto deCiências

Básicas da Saúde,

UniversidadeFederal do Rio Grande do Sul. Rua Sarmento Leite 500, Centro. 90050-170 Porto Alegre RS. fe.athayde@gmail.com ${ }^{2}$ Universidade do Vale do Rio dos Sinos.
}

Abstract Theantimicrobialsare one of thegroups of the most prescribed drugs in hospitalsand which cause a great concern for the suitability of their use. The profile of the antimicrobials utilized in a private hospital in the countryside of Rio Grande do Sul State was described and the quantity of each antimicrobial consumed was analyzed. All the prescriptions of patients who utilized antimicrobials while they were hospitalized in the period from M arch to June 2006 were analyzed. The antimicrobial consumption was expressed in Defined Daily Dose (DDD) in 100 day-beds. The antimicrobial utilization analysis were done generally speaking and divided by age groups and by medical clinics. During the studying period the antimicrobial use prevalencewas of $52.4 \%$ (IC 95 $\% 49.2$ - 55.7). The cephalosporinswere the most utilized ( $43.4 \%$ ), followed by thepenicillins ( 16.3 $\%)$, fluoroquinolones ( $13.0 \%$ ) and aminoglycosides $(9.7 \%)$. The high consumption of antimicrobials during the studying period is the result of the absence of a policy for controlling the drugs prescribed in hospital and the lack of protocols of antimicrobial use. In order to exist a control of antimicrobial consumption the hospitals must assume a surveillance policy on the prescriptions of this drug group.

Key words Antimicrobials, Drug utilization, Defined daily dose, Hospital prescription
Resumo Os antimicrobianos são um dos grupos de medicamentos mais prescritos nos hospitais e que causam uma grande preocupação quanto à adequação do seu uso. Descreveu-se o perfil dos antimicrobianos utilizados num hospital privado do interior do Rio Grande do Sul, analisando a quantidade consumida de cada antimicrobiano. Foram analisadas todas as prescrições de pacientes que utilizaram antimicrobianos enquanto internados no período de março a junho de 2006. 0 consumo dos antimicrobianos foi expresso em dose diária definida (DDD) por 100 leitos-dia. As análises de utilização de antimicrobianos foram feitas em geral e estratificadas por grupos de idade e por clínicas médicas. Durante o período em estudo, a prevalência do uso de antimicrobianos foi de $52,4 \%$ (IC 95\% 49,2-55,7). As cefalosporinas foram as mais utilizadas (43,4\%), seguidas das penicilinas (16,3\%), fluorquinolonas $(13,0 \%)$ e aminoglicosídeos $(9,7 \%)$. 0 alto consumo de antimicrobianos durante o período em estudo é resultado da ausência de uma política de controle dos medicamentos prescritos no hospital e da falta de protocolos de uso de antimicrobianos. Para que haja um controle do consumo de antimicrobianos, os hospitais devem assumir uma política de vigilância sobre as prescrições deste grupo de medicamentos.

Palavras-chave Antimicrobianos, Utilização de medicamentos, Dose diária definida, Prescrições hospitalares 
Introdução

As doenças infecciosas são combatidas com a utilização de antimicrobianos, sendo o seu uso adequado uma das principais preocupações mundiais ${ }^{1}$.

Essas drogas estão entre as mais frequentemente prescritas em hospitais. Cerca de $40 \%$ dos pacientes hospitalizados são tratados com antimicrobianos, tanto para indicações terapêuticas como profiláticas, eseu emprego inadequado tem proporcionado o surgimento cada vez maior de microorganismos resistentes ${ }^{2}$. Em trabalhos publicados sobre o uso de antimicrobianos nos Estados Unidos, foi constatado que mais da metade desse uso éinadequado ${ }^{3}$. O seu uso excessivo em hospitais contribui para o desenvolvimento de resistência bacteriana, aumentando os custos hospitalares e os riscos de reações adversas a medicamentos ${ }^{4}$. A resistência aos antimicrobianos éconsiderada atualmente como um grande problema de saúde pública, sendo de particular preocupação para os hospitais em função do aumento da morbimortalidade, além dos custos de saúde ${ }^{5}$.

0 aumento das taxas de resistência bacteriana não éum fenômeno recente, tendo a qualidade das prescrições um papel fundamental para preservar a efetividade dos fármacos antimicrobianos disponíveis. Cabe ressaltar a importância do papel dos profissionais da saúde para melhorar as condições atuais. Os antimicrobianos são os únicos medicamentos que influenciam não apenas o paciente, mas todo o ecossistema onde ele está inserido, com repercussões potenciais profundas $s^{6-8}$.

N os países em desenvolvimento, 0 uso indiscriminado é mais sério, pois normalmente estes produtos são de venda livre no comércio e as medidas de controle de uso em hospitais são inconsistentes. Os altos níveis de disponibilidadee consumo de antimicrobianos têm conduzido a um aumento desproporcional da incidência do uso inapropriado desses fármacos ${ }^{9}$. Segundo a Organização Mundial da Saúde (OM S), o uso inadequado dos medicamentos tem as seguintes características: prescrição em excesso, omissão da prescrição, doseinadequada, duração inapropriada, seleção inadequada, gasto desnecessário ou risco desnecessário $0^{9,10}$.

No âmbito hospitalar, prescritores com menor experiência clínica (internos eresidentes) tomam mais frequentemente as decisões terapêuticas e se sentem pressionados por casos agudos de alta complexidade ${ }^{10}$. A prioridade é evitar 0 desastre nas 24 horas seguintes, alvo suposta- mente alcançado com antibióticos de amplo espectro ou uso de vários antibióticos de pequeno espectro em associação. Outro fato comum é a repetição automática das prescrições, fazendo com que a duração de um curso de antibióticos se prolongue além do racional. Também a gravidade das infecções favorece a utilização de terapia empírica que pode levar à seleção de cepas resistentes ${ }^{9,10}$

0 impacto econômico da resistência bacteriana afeta de forma diferente os diversos atores envolvidos no processo de fornecimento e uso de antimicrobianos. 0 prescritor tem o custo da ineficácia da terapia convencional, com eventual perda de pacientes. 0 paciente tem o custo da doença não solucionada e de eventual morte, onerando-se com a exigência de medicamento alternativo. Por sua vez, o Sistema Público de Saúde gasta excessivamente, desequilibrando recursos geralmente escassos. Para a indústria farmacêutica, a resistência bacteriana é um incentivo para desenvolver novos fármacos. Já na "visão social", há uma redução de fonte de saúde (infecções mais graves, menos fármacos eficazes) para a populaçãa ${ }^{11}$.

Em função deste quadro, é degrandeinteressea real ização de estudos de utilização de antimicrobianos que permitam detectar problemas com os mesmos e realizar comparações em distintos âmbitos sanitários e localidades geográficas ${ }^{12}$. Estes estudos servem principalmente de subsídios para que os gestores possam revisar a política deantimicrobianos existentee avaliar sua aceitação e cumprimento ${ }^{3}$. A OM S preconiza o uso da metodologia ATC/DDD (The Anatomical The rapeutical Chemical Classification/D efined Daily Doses) como forma de padronizar a quantificação dos medicamentos utilizados, permitindo comparações de taxas de consumo através do tempo ${ }^{7,13,14}$.

Visando contribuir com a gestão do arsenal terapêutico intra-hospitalar, especificamente relacionado ao uso de antimicrobianos, e um controle da resistência bacteriana principalmentenos hospitais, este trabalho se propõe a descrever 0 perfil dos antimicrobianos utilizados num hospital privado, analisando as prescrições médicas destegrupo demedicamentos por clínica médica e faixa etária dos pacientes.

\section{Métodos}

Foi realizado um estudo transversal descritivo do perfil dos antimicrobianos utilizados em um 
hospital privado, em Santa M aria, no Rio Grande do Sul, no período de março a junho de 2006. A cidade de Santa M aria tem uma população residente de cerca de 270.000 mil habitantes ${ }^{15}$. 0 município é assistido por cinco hospitais, sendo dois de atendimento exclusivo ao SUS, dois de atendimento particular e aos planos de saúde e outro que presta atendimento particular, aos planos de saúde eao SUS. O hospital em estudo éde pequeno porte, com 72 leitos e está estruturado em unidades deinternação, bloco cirúrgico, unidade de tratamento intensivo (UTI) adulta, UTI pediátrica, UTI neonatal, unidade psiquiátrica e outras áreas de atendimento. A instituição presta atendimento somente a pacientes particulares e com planos de saúde.

No período estudado, o hospital ainda não possuía uma padronização definitiva de antimicrobianos, também não havendo um controle efetivo de restrição de uso de nenhum antimicrobiano utilizado, uma vez que a Comissão de Controle de Infecção H ospitalar estava em período de implantação.

Foram incluídas no estudo todas as prescrições dos pacientes internados no período de 01 de março a 30 de junho de 2006, que receberam pelo menos um tratamento com antimicrobiano sistêmico durante o período de internação. Os antimicrobianos de uso tópico foram excluídos do estudo pela dificuldade de dimensionar a quantidade recebida pelo paciente.

$\mathrm{Não}$ fizeram parte do estudo prescrições de antimicrobianos de pacientes ambulatoriais, já que estes, após os procedimentos realizados no hospital, eram encaminhados para seus domicílios, onde seguiam o tratamento.

Os dados foram coletados pela investigadora principal através da análise dos prontuáriose das informações do sistema informatizado existente no hospital - Sistema Integrado de Gestão H ospitalar (SIGH). As variáveis coletadas foram sexo, idade, clínica médica, antimicrobianos utilizados, doses, vias de administração, tempo de internação, duração do tratamento, número de pacientes internados no período e taxa de ocupação do hospital.

0 consumo dos antimicrobianos em todas as áreas clínicas da instituição foi expresso em dose diária definida (DDD) por 100 leitos-dia, por quatro meses. Utilizou-se a classificação ATC/ DDD da Organização Mundial de Saúde (OMS), versão 2006, para calcular o número deDDD para cada antimicrobiano utilizado ${ }^{13}$. A DDD constitui uma unidade de medida única, independente do preço e da forma farmacêutica, que permite avaliar as tendências no consumo de medicamentos. Em estudos no meio hospitalar, utiliza-se a DDD por 100 leitos-dia, que foi calculada a partir da seguinte fórmula:

DDD / 100 LEITOS/DIA = (quantidade de medicamento consumido $\times 100$ ) / (DDD estabelecida para o medicamento $x$ período de tempo observado em dias $x$ leitos disponíveis nas unidades do hospital $x$ índice de ocupação de leitos hospitalares no período estudado)

Os dados coletados foram registrados em planilha eletrônica do M icrosoft Office Excel 2003 eanalisados no pacoteestatístico STATA 8.0, onde foram calculadas médias, desvios padrão e as frequências absolutas erelativas das variáveis em estudo. As análises foram feitas em geral e estratificadas por grupos deidadee por clínicas médicas. As idades foram agrupadas da seguinte forma: um grupo de crianças e adolescentes ( 0 a 19 anos), um de adultos ( 20 a 49 anos) e dois grupos com idade mais avançada ( 50 a 69 e 70 anos ou mais), por ser o grupo mais frequente de pessoas internadas. As clínicas médicas apresentadas nos resultados são aquelas que apresentam maior proporção de antimicrobianos prescritos, sendo as demais especialidades agrupadas em um único grupo (outros). Os antimicrobianos foram classificados conforme a Anatomical Therapeutical Chemical (ATC) Classification Index ${ }^{13} \mathrm{em}$ oito grandes grupos farmacológicos (cefalosporinas, fluorquinolonas, penicilinas, aminoglicosídeos, derivados imidazólicos, glicopeptídeos, lincosamidas e novos beta-lactâmicos) e os menos frequentes foram agrupados em um único grupo (outros).

\section{Resultados}

Durante o período em estudo, 921 pacientes internaram-seno hospital e, desses, 483 fizeram uso de pelo menos um antimicrobiano, sendo a prevalência do uso de antimicrobianos de 52,4\% (IC95\% 49,2-55,7) e o número de prescrições de antimicrobianos, no período em estudo, de 866. As proporções do número de antimicrobianos no total de pacientes internados no período foram: $84,0 \%$ usaram até dois antimicrobianos; $11,8 \%$ usaram de três a quatro e 4,3\% usaram cinco ou mais antimicrobianos durante o período de internação. Dos antimicrobianos utilizados, $91,3 \%$ foram administrados pela via endove- 
nosa. Foram utilizados 36 antimicrobianos diferentes durante o período em estudo no hospital.

A idade dos pacientes variou de 0 a 100 anos, sendo a média de idade de 52,6 anos ( $D P=25,2$ ). 0 tempo de internação foi de 1 a 92 dias, sendo a média do tempo de internação de 14 dias (DP= $17,7)$ e a média de duração dos tratamentos de 5,6 dias ( $D P=6,3$ ), variando de 1 a 48 dias (mediana $=3$ ). A duração média de tratamento com antimicrobianos em função do período de internação foi de 2,2 dias ( $D P=1,39$ ) para os pacientes que permaneceram internados por até sete dias; de 8,1 dias ( $D P=5,07$ ) para o tempo de internação de oito a 30 dias e de 13,1 dias ( $\mathrm{DP}=9,51)$ para períodos de internação superiores a 30 dias.

A Tabela 1 indica as DDD s por 100 leitos-dia calculadas para cada um dos antimicrobianos utilizados no período em estudo.

A Tabela 2 demonstra o uso de antimicrobianos total e estratificado por faixas etárias dos pacientes internados. Entre os antimicrobianos utilizados, as cefalosporinas foram as mais frequentes $(43,4 \%)$, seguidas das penicilinas $(16,3 \%)$, fluorquinolonas (13,0\%) e aminoglicosídeos (9,7\%). Esses grupos foram responsáveis por mais de $80 \%$ dos antimicrobianos consumidos.

$\mathrm{N}$ as crianças e adolescentes ( 0 a 19 anos), os antimicrobianos mais utilizados foram as penicilinas, representando $1 / 3$ dos usos, seguido pelos aminoglicosídeos $(27,8 \%)$ e pelas cefalosporinas (22,1\%). Já na faixa etária dos 20 aos 49 anos, cerca de $60 \%$ dos usos foram de cefalosporinas, sendo o segundo grupo de antimicrobiano mais utilizado o das penicilinas (13,8\%). A partir dos 50 anos, os antimicrobianos mais utilizados também foram as cefalosporinas, só que em menores proporções, seguidos pelas fluorquinolonas e pelas penicilinas em segundo e terceiro lugares, respectivamente.

A Tabela 3 apresenta as proporções de antimicrobianos utilizados nos pacientes internados de acordo com as clínicas médicas. $\mathrm{Na}$ traumatologia, quase $60 \%$ das prescrições corresponderam ao grupo das cefalosporinas e os segundo e terceiro grupos de antimicrobianos mais utilizadosforam o dos aminoglicosídeos $(27,7 \%)$ efluorquinolonas $(7,6 \%)$, respectivamente. $\mathrm{Na}$ especialidade cirúrgica, mais de $50 \%$ das prescrições foram de cefalosporinas, seguidas das fluorquinolonas $(13,5 \%)$ e das penicilinas $(9,7 \%) . \mathrm{Na}$ gastroenterologia, 1/4 das prescrições corresponderam às penicilinas, sendo o segundo grupo mais prescrito o das cefalosporinas $(23,1 \%)$ seguidas das fluorquinolonas (16,5\%). Na gineco-
Tabela 1. Consumo de antimicrobianos de uso sistêmico, expresso em DDD (dose diária definida) por 100 leitos-dia, no período de março a junho 2006. Hospital Regional Unimed, Santa M aria (RS).

\begin{tabular}{|c|c|}
\hline Antimicrobianos utilizados & $\begin{array}{l}\text { DDD por } 100 \\
\text { leitos-dia }\end{array}$ \\
\hline Amicacina & 0,90 \\
\hline Ac. Clavulânico e Amoxacilina & 2,62 \\
\hline Amoxacilina & 0,43 \\
\hline Ampicilina & 1,58 \\
\hline Azitromicina & 1,05 \\
\hline Aztreonam & 0,49 \\
\hline Cefalotina & 2,82 \\
\hline Cefadroxil & 0,33 \\
\hline Cefalexina & 0,21 \\
\hline Cefazolina & 8,09 \\
\hline Cefepima & 1,32 \\
\hline Ceftazidima & 1,44 \\
\hline Cefotaxima & 0,18 \\
\hline Ceftriaxona & 7,95 \\
\hline Ciprofloxacino & 16,43 \\
\hline Claritromicina & 0,01 \\
\hline Clindamicina & 5,92 \\
\hline Eritromicina & 0,03 \\
\hline Ertapenem & 0,12 \\
\hline Gatifloxacino & 0,16 \\
\hline Gentamicina & 7,64 \\
\hline Imipenem + Cilastatina & 1,43 \\
\hline Levofloxacino & 0,32 \\
\hline Linesolida & 1,34 \\
\hline M eropenem & 1,38 \\
\hline Metronidazol & 5,13 \\
\hline Moxifloxacino & 1,63 \\
\hline Norfloxacino & 0,25 \\
\hline Oxacilina & 11,57 \\
\hline Penicilina G potássica & 3,59 \\
\hline Rifampicina & 0,26 \\
\hline Sulbactam + Ampicilina & 13,36 \\
\hline Sulfametaxozol + Trimetroprim & 0,15 \\
\hline Tazobactam + Piperacilina & 2,93 \\
\hline Teicoplanina & 0,83 \\
\hline Vancomicina & 3,96 \\
\hline
\end{tabular}

logia e obstetrícia, as cefalosporinas responderam por mais de $90 \%$ das prescrições. Na pediatria, os antimicrobianos mais utilizados foram as penicilinas (34,4\%), seguidas dos aminoglicosídeos (30\%) e das cefalosporinas (18,9\%). No grupo que englobou as demais especialidades, os antimicrobianos mais utilizados foram as cefalosporinas, com cerca de 1/3 das prescrições, seguidos das fluorquinolonas e penicilinas, com cerca de $20 \%$ das prescrições cada um. 
Tabela 2. Consumo de antimicrobianos de uso sistêmico nos pacientes internados de acordo com a faixa etária no período de março a junho de 2006. Hospital Regional Unimed, Santa M aria (RS).

\begin{tabular}{|c|c|c|c|}
\hline \multirow[b]{2}{*}{ Grupos de antimicrobianos } & \multicolumn{2}{|c|}{ Faixa etária (em anos completos) } & \multirow[b]{2}{*}{$\begin{array}{c}50-69 \\
N(\%)\end{array}$} \\
\hline & $\begin{array}{l}0-19 \\
N(\%)\end{array}$ & $\begin{array}{r}20-49 \\
N(\%)\end{array}$ & \\
\hline Aminoglicosídeos & $161(27,8)$ & $19(7,5)$ & $19(7,4)$ \\
\hline Cefalosporinas & $23(22,1)$ & $151(59,6)$ & $115(44,9)$ \\
\hline Derivados Imidazólicos & $5(4,8)$ & $8(3,2)$ & $10(3,9)$ \\
\hline Fluorquinolonas & $1(0,9)$ & $18(7,1)$ & $42(16,4)$ \\
\hline Glicopeptídeos & $7(6,7)$ & $7(2,7)$ & $9(3,5)$ \\
\hline Lincosamidas & $0(0,0)$ & $4(1,6)$ & $12(4,7)$ \\
\hline Novos beta-lactâmicos & $3(2,9)$ & $4(1,6)$ & $5(1,9)$ \\
\hline Penicilinas & $35(33,6)$ & $35(13,8)$ & $35(13,7)$ \\
\hline Outros & $1(0,9)$ & $7(2,8)$ & $9(3,5)$ \\
\hline Total & $104(100,0)$ & $253(100,0)$ & $256(100,0)$ \\
\hline
\end{tabular}

Tabela 3. Consumo de antimicrobianos de uso sistêmico nos pacientes internados de acordo com as clínicas médicas no período de março a junho de 2006. Hospital Regional Unimed, Santa M aria (RS).

\begin{tabular}{lrrrr}
\hline \multicolumn{1}{c}{ Antimicrobianos } & $\begin{array}{c}\text { Traumatologia } \\
\text { N (\%) }\end{array}$ & $\begin{array}{c}\text { Cirurgia } \\
\text { N }(\%)\end{array}$ & $\begin{array}{c}\text { Gastroenterologia } \\
\text { N }(\%)\end{array}$ & $\begin{array}{r}\text { Ginecologiae } \\
\text { obstetrícia } \\
\text { N }(\%)\end{array}$ \\
\hline Aminoglicosídeos & $33(27,7)$ & $5(4,8)$ & $4(4,4)$ & $2(1,8)$ \\
Cefalosporinas & $69(57,9)$ & $56(53,8)$ & $21(23,1)$ & $102(91,1)$ \\
Derivados imidazólicos & $1(0,8)$ & $4(3,8)$ & $13(14,3)$ & $2(1,8)$ \\
Fluorquinolonas & $9(7,6)$ & $14(13,5)$ & $15(16,5)$ & $2(1,8)$ \\
Glicopeptídeos & $2(1,7)$ & $3(2,9)$ & $3(3,3)$ & $1(0,9)$ \\
Lincosamidas & $1(0,8)$ & $7(6,7)$ & $3(3,3)$ & $0(0,0)$ \\
Novos beta-lactâmicos & $0(0,0)$ & $1(0,9)$ & $6(6,6)$ & $1(0,9)$ \\
Penicilinas & $4(3,7)$ & $10(9,7)$ & $23(25,3)$ & $2(1,8)$ \\
Outros & $0(0,0)$ & $4(3,8)$ & $3(3,3)$ & $0(0,0)$ \\
Totais & $119(100,0)$ & $104(100,0)$ & $91(100,0)$ & $112(100,0)$ \\
\hline
\end{tabular}

\section{Discussão}

0 presente estudo visou retratar o perfil do uso de antimicrobianos e possibilitou a identificação dos grupos mais utilizados, bem como das características gerais dos pacientes hospitalizados, contribuindo para o aprimoramento do controle desses medicamentos no âmbito hospitalar. Porém, problemas como dados de prontuários incompletos eausência de preenchimento dedados durante a internação foram fatores limitantes para que um estudo mais aprofundado pudesse ser realizado.
Estudos têm demonstrado que o uso de antimicrobianos nos hospitais tem sido considerado inapropriado, desnecessário ou excessivo ${ }^{6}$. Os microorganismos, que antes eram sensíveis aos antimicrobianos comuns e que se restringiam a causar infecções dentro do ambiente hospitalar, sendo a uma das principais causas de morbidade e mortalidade nos pacientes internados, mais recentemente estão se espalhando para a comunidade e causando doenças severas na população anteriormente saudável ${ }^{16}$.

O presente estudo encontrou uma prevalência de uso de antimicrobianos similar a de outro 
hospital brasileiro $(54,0 \%)$ de atendimento público, localizado no interior de São Paulo, com cem leitos, onde todos os pacientes adultos que usaram antimicrobiano foram avaliados durante um mês ${ }^{17}$. No entanto, foi mais baixa que a encontrada em um hospital de Israel $(62,0 \%)$, com 528 leitos, onde foram coletados dados de todos os antimicrobianos utilizados durante dois meses de verão e um ou dois meses de inverno ${ }^{18}$ etambém que a deum hospital do Peru (80,9\%), onde os pacientes em uso de antimicrobianos foram avaliados durante um mês ${ }^{6}$. Comparando-se com um estudo conduzido em outro hospital brasileiro (31,5\%), de atendimento privado, com 144 leitos, onde a coleta de dados foi realizada durante dezenove meses em pacientes adultos ${ }^{19}$, e com um estudo francês, realizado em um hospital universitário $(28,3 \%)$ de grande porte, com 1.228 leitos e com tempo de coleta de doze mese ${ }^{20}$, a prevalência foi bem mais alta.

0 alto consumo de antimicrobianos pode estar relacionado à ausência de uma política de controle dos medicamentos prescritos no hospital e à falta de protocolos de uso de antimicrobianos, gerando excesso de prescrições. As consequências do alto consumo de antimicrobianos, diferentemente de outros tipos de medicamentos, associado ao aumento de microorganismos resistentes, afetam não somente o paciente que está com a infecção mas também o paciente internado na cama ao lado, ou mesmo pacientes que já estiveram internados no hospital e que levam os microrganismos resistentes para a comunidade. Com isso, podem ser desencadeadas infecções que requerem tratamentos mais complexos e onerosos, gerando maiores gastos com saúde, que vão desde uma maior permanência do paciente no hospital, englobando gastos com exames mais sofisticados e procedimentos, utilização de antimicrobianos mais potentes até gastos indiretos representados pela perda de produtividade do paciente ${ }^{5,21,22}$.

A maior parte dos antimicrobianos foi administrada pela via endovenosa, o que já era esperado em função das condições físicas dos pacientes internados; entretanto, foi superior aos achados de outro estudo asiático $(64,0 \%)^{18}$, que avaliou um total de 6.376 antimicrobianos indicados no período estudado e aos de um estudo europeu $(69,0 \%)^{23}$, queanalisou 657 antimicrobianos prescritos. É importante salientar que a via endovenosa é mais onerosa, além de ser mais uma porta de entrada para infecções hospitalares. Dessa forma, sempreque os níveis sanguíneos do antimicrobiano forem equivalentes na via oral e endovenosa e o paciente estiver em condições de ingeri-Io, o prescritor deveria fazer a conversão para via oral, já que esta, além de mais segura contra infecções, é também mais econômica para o hospital ${ }^{5}$.

No presente estudo, a maior parte dos pacientes internados queutilizaram antimicrobianos receberam até dois medicamentos e uma pequena parcela recebeu cinco ou mais. Estas proporções de número de antimicrobianos por paciente estão de acordo com outro estudo realizado no interior do Rio Grande do Sul em outro hospital privado com 144 leitos, onde dos 1.166 pacientes que usaram antimicrobianos, 92,6\% utilizaram até dois medicamentos, 5,8\% utilizaram de trêsa quatro e $1,5 \%$ utilizou cinco ou mais medicamentos ${ }^{19}$.

A média do tempo deinternação foi um pouco superior às médias encontradas em outros estudos, que variaram de 5,5 a 11,2 dias 17,19,24. A diferença entre as médias está relacionada ao tipo de paciente atendido em cada hospital, às especialidades médicas disponíveis e aos tratamentos executados, podendo oscilar em diferentes períodos do ano de acordo com as causas da internação.

A média de dias de tratamento com antimicrobianos no presente estudo foi similar à observada em outra pesquisa de avaliação de uso deantimicrobianos, realizada durantetrês a quatro meses em dezenove unidades de tratamento em um hospital de grande porte de I srael ${ }^{18}$.

Foram calculadas as DDDs para cada antimicrobiano utilizado no período da coleta de dados com o objetivo de servir para futuros estudos comparativos intra-hospitalares. Optouse por calcular cada medicamento em separado para que houvesse uma melhor visualização do que foi consumido pelo hospital. A maior parte dos estudos utilizando a metodologia DDD realizam os cálculos por grupos de antimicrobianos $^{1,3,8,14,25}$. Somente dois estudos foram localizados calculando as DDDs para cada um dos antimicrobianos consumidos ${ }^{14,24}$, sendo queum deles calculou o quefoi consumido nas unidades de tratamento intensivo em separado das outras clínicas ${ }^{12}$. O btivemos valores maiores de DDD para o ciprofloxacino $(16,43)$, sulbactam associado à ampicilina $(13,36)$, oxacilina $(11,57)$ em comparação com um estudo italiano ${ }^{23}$, onde todas as DDDs foram bem mais baixas, sendo os antimicrobianos com maiores DDDsa netilmicina $(7,23)$, a ceftriaxona $(3,61)$ e a ceftazidima $(2,44)$. Já em comparação com um estudo espanhol ${ }^{12}$, em quea cefepima $(46,47)$, a gentamicina $(22,92)$ e a tobramicina $(22,62)$ foram as maiores DDDs, os valores foram maiores que os en- 
contrados em nosso estudo, deixando claro que cada hospital tem suas próprias características e que tanto o tipo de antimicrobiano quanto a quantidade consumida é bem diferenciada, pois envolve uma série defatores locais que contribuem para prescrições preferenciais de um ou outro antimicrobiano. A metodologia ATC/DDD foi criada para permitir comparações entre os medicamentos utilizados; entretanto, devemos levar em conta que cada local de estudo tem suas próprias características e essas diferenças devem ser consideradas para que as comparações tenham significado prático. Já as comparações feitas com dados do mesmo hospital, em diferentes períodos, podem servir de indicativo da tendência de uso de medicamentos.

Com relação à proporção de cada antimicrobiano utilizado, o grupo das cefalosporinas foi o mais consumido, o que pode ter sido influenciado pelo grande número de tratamentos profiláticos em pacientes cirúrgicos. 0 segundo grupo mais consumido foi o das penicilinas, sendo essa proporção também verificada em dados do Ministé rio da Saúde em relação à utilização dos grupos de antimicrobianos em hospitais brasileiros prestadores de serviços para o SUS ${ }^{26}$, embora o hospital em estudo não preste esse tipo de atendimento.

As terceiras e quartas mai ores proporções de antimicrobianos (fluorquinolonas eaminoglicosídeos) diferem tanto dos dados do Ministério da Saúde quanto de um estudo desenvolvido em um hospital de Porto Alegre ${ }^{1}$, que encontraram os aminoglicosídeos como o terceiro antimicrobiano mais utilizado. Esta diferença pode ocorrer pela grande quantidade de infecções graves no período em estudo, onde o ciprofloxacino costuma ser amplamente utilizado; no entanto, para que esses dados fossem melhor analisados, seria importante classificar os antimicrobianos quanto à indicação terapêutica, o que não foi possível, pois tanto os prontuários quanto o Sistema Integrado de Gestão H ospitalar tinham informações incompletas, além defrequentemente a evolução médica do pacienteser preenchida após esse receber alta hospitalar.

Outro fator limitante foram os exames microbiológicos que eram entregues e armazenados nos prontuários muito tempo após a sua realização, o que impossibilitou a identificação das causas de troca ou suspensão dos medicamentos utilizados, impossibilitando que fossem feitas análises de adequação de doses de antimicrobianos.

0 grupo das cefalosporinas foi o mais utilizado em todas as faixas etárias, exceto na faixa dos 0 19 anos, em que as penicilinas foram as mais utili- zadas. Da mesma maneira, como foi descrito em um outro estudo, a maioria das fluorquinolonas foram prescritas para os pacientes mais idosos?

O número de pacientes idosos internados foi bastante expressivo, já que seguidamente essas pessoas necessitam de internação hospitalar para cuidar de suas condições clínicas. O s idosos são mais suscetíveis a adquirir infecções que 0 adulto jovem devido a alterações fisiológicas do enveIhecimento, além de procedimentos invasivos ${ }^{27,28}$. Além disso, possuem reduzida capacidade de reagir a infecções, sendo necessário um cuidado especial na escolha dos antimicrobianos, já que estes interagem com muitos medicamentos que frequentemente fazem parte dos tratamentos destes pacientes ${ }^{27}$.

As clínicas médicas traumatologia, ginecologia e obstetrícia e cirurgia apresentaram mai ores proporções de prescrições de cefalosporinas, demonstrando o grande uso de cefalosporinas de primeira geração de uso profilático nesses pacientes devido a sua boa eficácia ebaixa toxicidade, sendo os esquemas profiláticos com cefazolina ou cefal otina bastante recomendados ${ }^{27}$.

$\mathrm{Na}$ especialidade cirúrgica, também fizeram parte pacientes que já tinham ou adquiriram infecções no hospital e foram tratados com cefalosporinas de terceira geração que são recomendadas em esquemas terapêuticos para infecções mais graves e preferencialmente em infecções hospitalares ${ }^{27}$. $\mathrm{Na}$ traumatologia, as cefalosporinas são também usadas em profilaxia de colocação de próteses ${ }^{27}$, que foram frequentes durante o período em estudo.

$\mathrm{Na}$ gastroenterologia, a classe de antimicrobianos predominante foi a das penicilinas, que estão presentes em muitos esquemas de tratamento deinfecções do trato gastrointestinal, também fazendo parte dos esquemas profiláticos ${ }^{27}$.

$\mathrm{N}$ a pediatria, as penicilinas foram os antimicrobianos mais utilizados, que são medicamentos presentes em muitos esquemas de tratamento de doenças infecciosas respiratórias, do trato gastrointestinal, sistema locomotor e sistema nervoso centra|27, que são os tipos de infecções mais atendidas no hospital em estudo. Cabe ressaltar que a terapêutica antimicrobiana em pediatria apresenta características próprias por que, além dos patógenos infecciosos prevalentes variarem com as faixas etárias, a farmacocinética das drogas é afetada pelas variações da composição corporal e pela maturação dos sistemas de metabolismo e excreção $0^{27}$.

0 hospital onde foi realizado o estudo, embora de pequeno porte, possui unidades que re- 
querem utilização de antimicrobianos tanto de forma terapêutica quanto profilática, sendo de fundamental importância identificar a proporção de antimicrobianos que está sendo consumida equais podem estar sendo utilizados deforma indevida. Apesar de não ser possível comparar a gravidade dos casos atendidos com a de outros hospitais, uma vez que cada um atende um tipo característico depacientes eutiliza medicamentos indicados para atender a sua comunidade, a metodologia empregada utiliza uma unidade internacional de medida que tem um significado de consumo independente de outros fatores.

0 estudo da utilização de antimicrobianos é relevante também em termos econômicos, uma vez que estes medicamentos respondem por um dos grupos em que os gastos hospitalares com medicamentos são maiores. Avaliações precisas dos custos com infecções adquiridas no hospital têm implicações importantes para os pacientes, gestores e pagadores. Para convencer os gestores hospitalares e de sistemas de saúde a investir em prevenção deinfecções, énecessário que setenha dados confiáveis de mortalidade atribuída, prolongamento da permanência no hospital e custos adicionais com atendimentos, exames, tratamentos e materiais médico-hospitalares causados pelas infecções hospitalares. Decisões a respeito da extensão de recursos hospitalares devo- tados ao controle das infecções e ao plano de incentivos para o hospital reduzir a taxa de infecções requerem informações precisas fornecidas pelo hospital através dos programas de controle de infecção hospitalar e do controle dos antimicrobianos em uso ${ }^{28,29}$. A vigilância constante das infecções hospitalares e o desenvolvimento de políticas apropriadas para o controle do uso de antimicrobianos são de alta prioridade ${ }^{30}$.

Considerando que os antimicrobianos são medicamentos de grande importância e que têm uma frequência elevada de utilização, é preciso urgentementedesenvolver políticas de saúdeque priorizem o seu uso de forma racional. Os hospitais devem ter um controle das prescrições através de uma comissão de controle de infecção hospitalar, proporcionando uma educação continuada aos profissionais da saúde.

Além disso, percebe-se a necessidade de mais estudos de utilização de antimicrobianos que avaliem doses prescritas, interações envolvendo esses medicamentos e identificação de reações adversas, através da análise de prescrições, adequação de tratamentos e profilaxia. Também são fundamentais estudos defarmacovigilância, uma vez que esses medicamentos são essenciais para o tratamento das doenças infecciosas e têm uma influência incontestável na ecologia microbiana, sendo o seu uso inadequado nocivo para todos.

\section{Colaboradores}

FA Rodrigues foi responsável pela elaboração do projeto que originou este trabalho, revisão de literatura e definição dos objetivos do estudo. Real izou a coleta das informações e também participou das análises eredação do manuscrito. AD Bertoldi participou na supervisão do projeto, elaboração das análises e redação final do artigo. 


\section{Referências}

1. Castro MS, Pilger D, Ferreira M BC, Kopittke L. Tendências na utilização de antimicrobianos em um hospital universitário, 1990-1996. Rev Saude Publica 2002; 36(5):553-558.

2. Bolufer JVA, Montero CT. Estúdio de la utilización de antibióticos de um hospital comarcal. Años 19982002. Farm Hosp (M adrid) 2004; 28(6):410-418.

3. Rosa MB, Reis AMM, Lima CR. A Farmácia e 0 controle das infecções hospitalares. In: Gomes MJVM, Reis AM M, organizadores. Ciências farmacêuticas : uma abordagem em farmácia hospitalar. São Paulo: Atheneu; 2003. p. 407-427.

4. Fuchs FD. Princípios gerais do uso de antimicrobianos. In: Fuchs FD, Wannmacher L, Ferreira MBC, organizadores. Farmacologia clínica: fundamentos da terapêutica racional. $3^{a}$ ed. Rio de Janeiro: Guanabara Koogan; 2004. p. 342-359.

5. Philmon C, Smith T, Williamson S, Goodman E. Controlling Use of Antimicrobials in a Community Teaching Hospital. Infect Control Hosp Epidemiol 2006; 27:239-244.

6. Maldonado FC, Zavalaga FL, Mayca JP. Uso y prescripción de medicamentos antimicrobianos en el hospital de apoyo de la Merced - Perú. Rev. perú med. exp. salud publica 2002; 19(4):181-185.

7. Natsch $S$, Hekster $Y A$, de Jong $R, H$ eerdink $E R$, Herings RM C, van der M eer JWM. Application of the ATC/ DDD methodology to monitor antibiotic drug use. Eur J Clin Microbiol Infect Dis 1998; $17(1): 20-24$.

8. Plascencia LB, Ojeda ALA, Vázquez HJ. Vigilancia de los niveles de uso de antibióticos y perfiles de resistencia bacteriana en hospitales de tercer nivel de la ciudad de M éxico. Salud Publica M ex 2005; 47(3):219-226.

9. World Health Organization. Interventions and strategies to improve the use of Antimicrobials in developing countries: a review. [site da Internet] [acessado 2006 dez 15]. Disponível em: http://www.who.int/ drugresistance/Interventions_and_strategies_to improve the use_of_antim.pdf

10. Wannmacher L. Uso indiscriminado de antibióticos e resistência microbiana: uma guerra perdida? U so racional de medicamentos: temas selecionados 2004; $1(4): 1-6$.

11. França FB, Costa AC. Perfil farmacoterapêutico de pacientes em uso de antimicrobianos em hospital privado, em Fortaleza - CE. RBPS 2006; 19(4):224228.

12. Nájera LH, Blasco AC, Sanz UM, Osinaga EA, Inchaurregui LCA. Evolución de la utilización de antimicrobianos durante los años 1996-2000 em um hospital general. Estudio pormenorizado de la $\mathrm{UCl}$. Farm Hosp (M adrid) 2003; 27(1):31-37.

13. WHO Collaborating Centre for Drug Statistics Methodology. Anatomical Therapeutic Chemical (ATC) Classification and Defined Daily Doses (DDD).[site da Internet]. [acessado 2006 jun 09]. Disponível em: http: //www.whocc.no/atcddd/atcsystem.html

14. Filius PM G, Liem TBY, van der Linden PD, Janknegt $R$, Natsch S,Vulto AG, Verbrugh HA. An additional measure for quantifying antibiotic use in hospital. J Antimicrob Chemother 2005; 55:805-808.
15. Brasil. Ministério da Saúde. DATASUS. População residente - Rio Grande do Sul [site da Internet]. [acessado 2006 dez 05].Disponível em: http:// tabnet.datasus.gov.br/cgi/tabcgi.exe?ibge/cnv/ popRS.def

16. Alanis AJ. Resistance to Antibiotics: Are We in the Post- Antibiotic Era? Arch M ed Res 2005; 36(6):697705.

17. Fonseca LG, Conterno LO. Audit of antibiotic use in Brazilian university hospital. BJID 2004; 8(4):272280.

18. Raveh D, Levy Y, Schlesinger A, Greenberg B, Rudensky $B$, Yinnon AM. Longitudinal surveillance of antibiotic use in the hospital. Q J M ed 2001; 94:141-152.

19. Spiandorello WP, Faintuch J, Ribeiro GT, Karkow FJ, Alvares JO. Use of multiple antimicrobial drugs by clinical patients: a prognostic index of hospital mortality? Clinics 2006; 61(1):15-20.

20. Muller $A$, Monnet $D L$, Talon $D$, Hénon $T$, Bertrand $X$. Discrepancies between prescribed daily doses and WHO defined daily doses of antibacterials at a university hospital. Br J Clin Pharmacol. 2006; 61(5):585-591.

21. Larson E, Quiros D, Giblin T, Lin S. Relationship of Antimicrobial Control Policies and Hospital and Infection Control Characteristics to Antimicrobial Resistance Rates. Am J Crit Care 2007; 16(2):110-120.

22. Goldman MP, Nair R. Antibacterial treatment strategies in hospitalized patients: What role for pharmacoeconomics? Cleve Clin J M ed 2007; 74(4):38-47.

23. M andy $B$, Koutny $E$, Cornette $C$, Wonoroff-Lemsi MC, Talon D. Methodological validation of monitoring indicators of antibiotics use in hospitals. Pharm World Sci 2004; 26:90-95.

24. Basseti M, Di Biagio A, Rebesco B, Amalfitano ME, Topal J, Bassetti $D$. The effect of formulary restriction in the use of antibiotics in an Italian hospital. Eur J Clin Pharmacol. 2001; 57:529-534.

25. Vlahovic-Palcevski V, Morovic M, Palcevski G. Antibiotic utilization at the university hospital after introducing an antibiotic policy. Eur J Clin Pharmacol. 2000; 56:97-101.

26. Prade SS, Oliveira ST, Rodriguez $R$, Nunes FA, Netto EM, Felix JQ, Pereira M, Gadelha MZ, Borba EA, $M$ endes $A$. Estudo brasileiro de magnitude das infecções hospitalares em hospitais terciários. Rev Cont Inf Hosp. 1995; 2:11-25.

27. Barros E, Bittencourt $H$, Caramori ML, Machado A. Antimicrobianos: consulta rápida. 3a ed. Porto Alegre: Artmed; 2001.

28. Villas Boas PJF, Ruiz T. Ocorrência de infecção hospitalar em idosos internados em hospital universitário. Rev. Saude Publica 2004; 38(3):372-378.

29. Kilgore ML, Ghosh K, Beavers M, Wong DY, Hymel PA Jr, Brossette SE. The costs of nosocomial infections. M ed Care 2008; 46:101-104.

30. Gastmeier P. Nosocomial infection surveillance and control policies. Curr O pin Infect Dis 2004; 17:295301.

Artigo apresentado em 09/07/2007 Aprovado em 11/02/2008 